\title{
Lane Detection by Orientation and Length Discrimination
}

\author{
Andrew H. S. Lai, Member, IEEE, and Nelson H. C. Yung, Senior Member, IEEE
}

\begin{abstract}
This paper describes a novel lane detection algorithm for visual traffic surveillance applications under the auspice of intelligent transportation systems. Traditional lane detection methods for vehicle navigation typically use spatial masks to isolate instantaneous lane information from on-vehicle camera images. When surveillance is concerned, complete lane and multiple lane information is essential for tracking vehicles and monitoring lane change frequency from overhead cameras, where traditional methods become inadequate. The algorithm presented in this paper extracts complete multiple lane information by utilizing prominent orientation and length features of lane markings and curb structures to discriminate against other minor features. Essentially, edges are first extracted from the background of a traffic sequence, then thinned and approximated by straight lines. From the resulting set of straight lines, orientation and length discriminations are carried out three-dimensionally with the aid of two-dimensional (2-D) to three-dimensional (3-D) coordinate transformation and $K$-means clustering. By doing so, edges with strong orientation and length affinity are retained and clustered, while short and isolated edges are eliminated. Overall, the merits of this algorithm are as follows. First, it works well under practical visual surveillance conditions. Second, using $\boldsymbol{K}$-means for clustering offers a robust approach. Third, the algorithm is efficient as it only requires one image frame to determine the road center lines. Fourth, it computes multiple lane information simultaneously. Fifth, the center lines determined are accurate enough for the intended application.
\end{abstract}

Index Terms-Camera parameter estimation, center line detection, edge detection, lane detection.

\section{INTRODUCTION}

I $\mathrm{T}$ IS commonly believed that the smooth operations of future intelligent transportation systems (ITS) rely heavily on the amount of vehicle and road information acquired. At present, different sensors have been deployed for this purpose, e.g., buried loop detectors for detecting vehicle counts and signatures, radar/laser sensors for tracking vehicle speed, and video cameras for general surveillance. Moreover, other electronic and optical sensors have also been developed for detecting weight, vehicle registration, surface friction, presence of vehicles, etc. [1]. Among those, video or CCTV technology presents an exciting option as they can potentially extract

Manuscript received September 16, 1998; revised March 12, 2000. This paper was supported by the University Grants Committee, Hong Kong, by the Area of Excellence in Information Technology under Grant AOE98/99.EG01, by the Committee on Research and Conference Grant of the University of Hong Kong, by the research postgraduate studentship, and by the Li Po Chun Scholarship. This paper was recommended by Associate Editor P. Willett.

The authors are with the Laboratory for Intelligent Transportation Systems Research, The University of Hong Kong, Pokfulam, Hong Kong.

Publisher Item Identifier S 1083-4419(00)06714-5. a rich set of information in the most unobtrusive manner compared with the other sensors. The argument is that from video sequences taken from roadside or overhead mounted cameras, valuable information such as travel time, speed, count, queue length, and class may be estimated using an image/video processing technique [2]. However, the pitfalls of employing image/video processing in visual traffic surveillance is that it is susceptible to effects created by natural outdoor phenomena such as day-night changes, shadows, rain, fog, dust, etc. Even under a normal and benign outdoor environment, current image/video processing techniques do not guarantee robust solutions.

One of the major challenges in surveillance is to detect the presence of vehicles and their trajectories (speed and direction) from which the estimation of other parameters and conditions may be based. To do so, motion estimation and vehicle modeling are two typical approaches reported in literatures [3]-[5]. Usually, lane direction is assumed known and used for refining motion estimation and tracking [6], or not needed in cases where the viewing angle is not along the lane direction. However, if further information such as driver characteristics (how well a vehicle follows the lane) and lane change traits (how often a vehicle changes lanes) are to be determined, our knowledge of lane direction and center lines becomes crucial. Although there are sensors that can detect the camera's tilt angle and distance from the vehicle, which can roughly then estimate the lane direction, they are mostly inaccurate and inadequate to determine the characteristics as described above. As such, we are motivated to find a robust lane detection algorithm that can accurately extract complete multiple lane information including the center lines from an image or a video sequence.

The goal of lane detection in this case is to locate the center line of each lane from a multilane digital road image. To achieve this goal, lane markings may be used to differentiate the lanes from other features such as trees, bushes, humans, and others that may possibly be in the image. The major difficulties in detecting the lane markings correctly are that first, they are not always clearly visible due to their print quality and the changes in environmental conditions. Second, the geometry of the markings cannot be used as a discriminating factor as there is no governing standard. Further, road splitting or merging and the interference from roadside objects or shadows could worsen the detection. In this paper, we propose a novel lane detection algorithm that is capable to deal with the above difficulties. As long as the lane markings have reasonable print quality and the roadside curb structures can be seen, environmental changes, variation in marking geometry, splitting, merging, roadside objects, and shadows can all be tolerated in the algorithm. In principle, it employs an edge-based approach to extract a set of edge features 
of all the objects in the image, which include the lane markings, curbs, roadside objects, and many other meaningful or meaningless features. These edges are then thinned and approximated by straight lines. From the resulting set of straight lines, orientation and length discriminations are carried out three-dimensionally with the aid of two-dimensional (2-D) to three-dimensional (3-D) coordinate transformation and $K$-means clustering. The basis for this approach is that edge lines describing the lane markings and curbs are long and are almost parallel in the 3-D space. Given a correct clustering condition, these lines will cluster together, while other short and isolated edge lines will cluster into a different class, which can subsequently be discarded. From the lane/curb edge lines, center lines of lanes can be determined. Overall, the merits of this algorithm are as follows. First, it works well under practical visual surveillance conditions. Second, using $K$-means for clustering offers a robust approach. Third, the algorithm is efficient as it only requires one image frame to determine the center lines. Fourth, it computes multiple lane information simultaneously. Fifth, the center lines determined are accurate enough for the intended application.

This paper is organized as follows. Section II outlines some of the previous works in region-based and edge-based lane detection. Section III gives an overview of the proposed solution. Section IV briefly describes the edge detection, while Section V discusses how the camera parameters are estimated and being used in the 2-D to 3-D coordinate transformation. Section VI details the heuristics used for orientation and length discrimination and Section VII describes the lane analysis. Section VIII evaluates the algorithm using a practical freeway image, and the paper is concluded in Section IX.

\section{Previous WORK}

Over the years, many road lane detection methods have been developed for vehicle navigation. Algorithms reported so far [7]-[17] all focused on detecting segments of single lane road markings from an on-vehicle camera, using spatial masks to isolate the left and right lane markings. In some cases, the perspective image mask is transformed into 3-D before heuristics are applied to identify regions defining the lane, whereas in others, edge detection is applied to multiple window strips directly. When processing the masks, region-based and edge-based methods are both popular. From the results, the road center line is then determined. The following paragraphs describe some of these methods.

For instance, Schaaser and Thomas [7] employed a region-based method to segment road markings and modeled them by circular arcs. Two markings are linked together if they are concentric. The linked arcs are approximated by second-order quadratic curves that described the road boundaries. As only circular arcs are used for modeling the road markings, this method lacks flexibility. Moreover, the modeling and linking complexity increased exponentially with the number of dashed markings per lane boundary, which is worse for multiple-lane detection.

Jochem and Baluja [8] used the Hough Transform to extract road boundaries from an image, where the extracted boundaries are classified into road or nonroad regions by a single trained perceptron, which required extensive training before it can be used. From the road regions, they relied on a trapezoidal road model to determine the center lines. Due to its computing complexity, parallel computation is a basic requirement for this algorithm.

Thorpe et al. [11] proposed a different region-based approach for road detection in the Navlab. They assumed that the road has no road markings at all. Image pixels are classified into road and nonroad pixels according to their colors, based on known road colors. Naturally, any change in outdoor illuminations may change the road colors perceived by the camera and introduce errors in the classification. This method works well on a road image with good contrast between the road and roadside objects. Whether it works for other type of images with roadside objects having similar colors to the road colors is yet to be seen.

Kasprzak et al. [12] presented a road parameter estimation method which classifies edges into three classes. Vanishing point is used to define the horizontal ground. Edges above the horizontal ground are eliminated while edges below the horizontal ground are further classified as road and nonroad edges. The stability of the algorithm seems to be highly dependent on the vanishing point detection while the accuracy of the detection is generally low and depending on the image.

Tang and Kasturi [13] developed a runway detection algorithm for aircraft. They used Canny's edge detection to extract edges from the image. These edges are classified into straight edges, circular arcs, and various types of junctions, which are then described by marking models to form the basis of recognition. The algorithm enjoys certain success because runways usually have very clear and well-defined markings.

Kluge and Lakshmanan [15] described a deformable template approach that is based on an image intensity gradient. A likelihood function was used to provide a relative measurement of how well a given set of shape parameters matched the pixels. The algorithm uses an iterative method in which given sufficient number of iterations have been performed; the detected road matches closely with the actual road. However, the number of iterations required to achieve this can be up to 703000 , as mentioned in their paper.

In summary, most of the region-based methods used road markings to differentiate the road regions from the other nonroad regions. They can only handle one lane, and the images must fit under a set of conditions, such as illumination, color, and contrast, which limits the application of them in visual surveillance. For the edge-based methods, they employ sophisticated techniques on top of edge detection to improve the quality of the detection. Unfortunately, these techniques either incur heavy computing overhead or the environment has to be very well defined. It is also observed that other useful criteria are seldom considered. For instance, roadside curbs usually give a fairly good description of the road boundary and direction and are consistent with the lane markings, but have not been taken into account. Therefore, for surveillance, these features can potentially be utilized as additional parameters in differentiating the road/lane edges from nonroad edges.

\section{OVERVIEW OF SOLUTION}

From the above observations, our solution is based on edge detection and the discrimination of edge lines by orientation and length. As depicted in Fig. 1, the road image may be acquired 


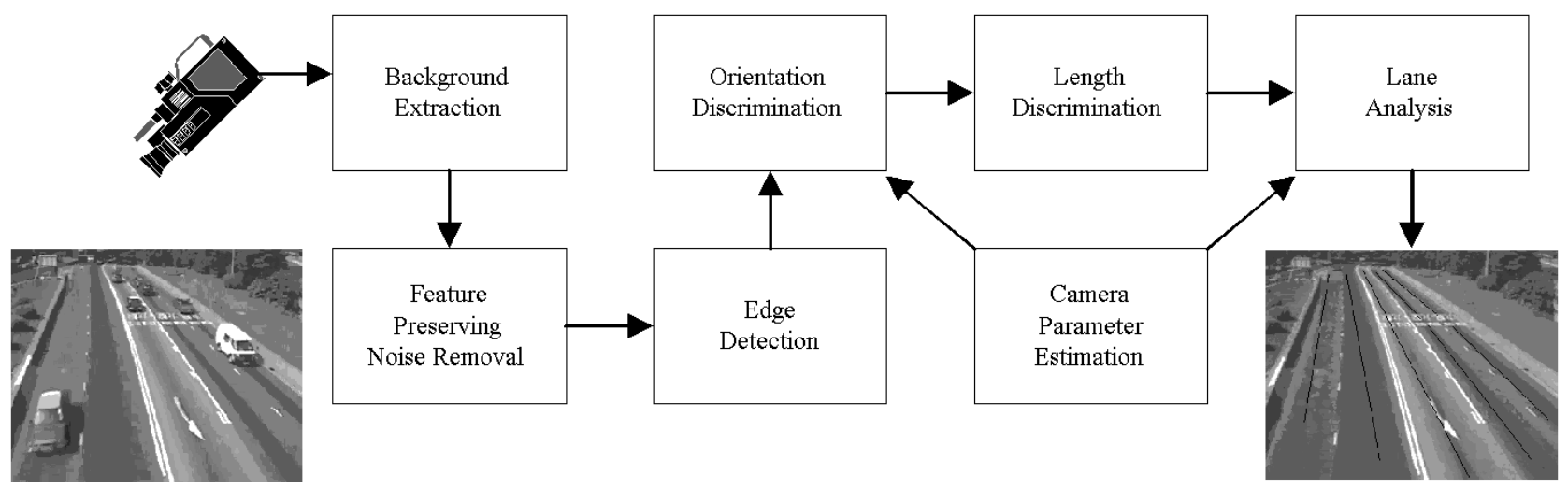

Fig. 1. Block diagram of the lane detection algorithm.

from a still or video camera. For the still image, it has to be taken when there are no vehicles on the road. For video, a normal sequence with moving vehicles may be used from which the background can be estimated. In this research, the road image was determined from a normal traffic video with moving vehicles through background estimation [16], using a small number of frames. Once the background has been estimated, feature preserving filtering is employed to remove additive white noise due to quantization or transmission [17], [18] to minimize the blurring of edges due to filtering. Then, edges are extracted, thinned, and approximated by straight lines where each line has an associated orientation and length [19].

These edge lines are first clustered according to their orientation using the $K$-means clustering technique. As images taken from roadside cameras are usually in a 2-D perspective view, orientation clustering must be done in 3-D to make sense. To perform this transform, camera parameters including focal length, mounting height, and its distance to the focused point on the road must be known. When viewed from the $z$-axis, edges of lane markings and curbs appear to be parallel and will cluster as a result. In contrast, edges of roadside objects, uneven road color or surfaces, or noise, are dissimilar in orientations, which may be discarded. From the orientation discrimination results, edge lines are linked and clustered according to their length using the $K$-means technique again. The purpose of linking is to connect those short edge lines with similar orientations, which could be part of a broken line or a poorly detected solid line. The clustering further isolates the short edge lines, which may be discarded. Up to this point, it is possible that some of the lane markings are poorly detected and, therefore, missing in the resulting edge set. However, curb features may be relied upon as they are usually prominent in most road images. For lane analysis, center lines are calculated based on an assumed road width and the separation between two detected lines adjacent to each other. As it is, the approach is capable to estimate missing lanes in multilane cases.

\section{EDGE DETECTION}

As depicted in Fig. 2, lane markings are either solid or broken lines painted white or yellow. Other markings such as direction arrows and instructions painted on the road are also common. In addition, slightly elevated curb structures are often used to sep-

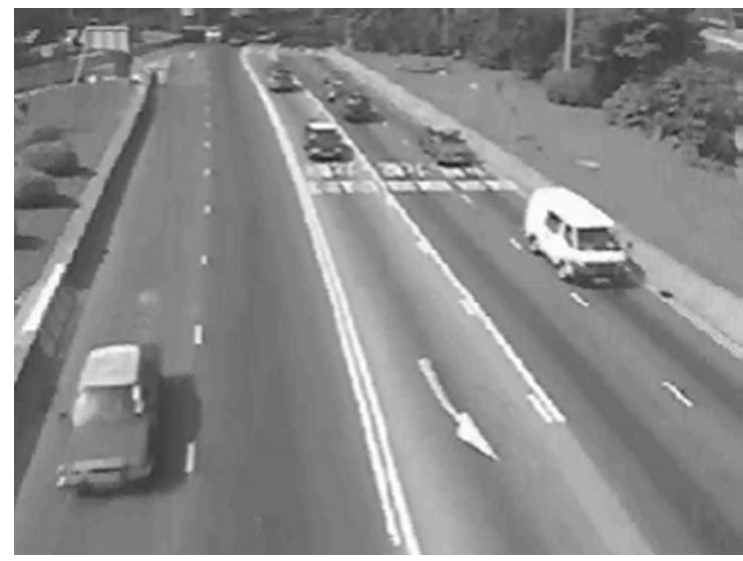

(a)

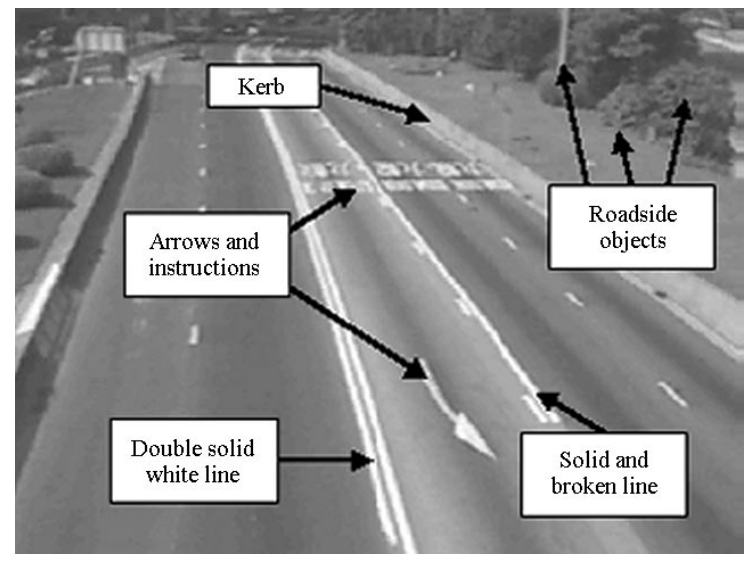

(b)

Fig. 2. City road. (a) Original image. (b) Extracted background.

arate roadside objects such as bushes, lampposts, and buildings from the road. To detect all these edges, a reliable and accurate edge detector is preferred, as further discriminations will eliminate the unwanted ones. In this case, the Sobel edge detector was chosen for its differencing and smoothing property [19]. The edge map is thinned as shown in Fig. 3(a). As can be seen in Fig. 3(a), local color variations in the image are all detected as edges, e.g., the white spots on the leftmost lane, the different surface color of the lane right of the double white line, and the instructions on the road. The edge pixels are then linearly approximated into straightline segments as depicted in Fig. 3(b). As a 


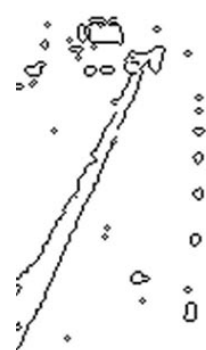

0

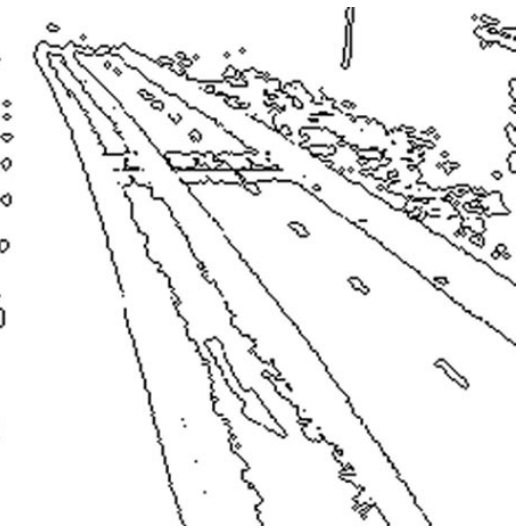

(a)

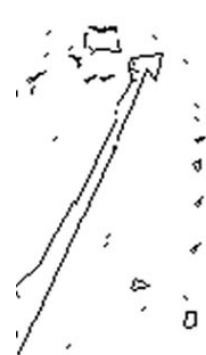

$\nabla$

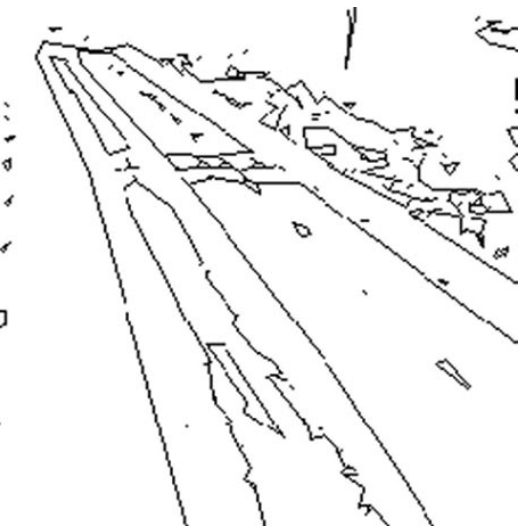

(b)

Fig. 3. Edge maps of Fig. 2. (a) Thinned-edge map. (b) Straight-line approximated edge map.

result, the approximated edges become smoother and some of the small local edge variations have now disappeared, although the edge map remains complex.

Mathematically, each edge point is approximated by $\left|\mathbf{G}_{\mathbf{x}}\right|+$ $\left|\mathbf{G}_{\mathbf{y}}\right|$. Given an image $I$, the set of edge lines $E$ is defined as a collection of straight lines satisfying the following:

$$
E=\left\{e_{k}=\left(p_{k}\left(x_{h}, y_{h}\right), p_{k}\left(x_{t}, y_{t}\right)\right)\right\}
$$

where $p_{k}\left(x_{h}, y_{h}\right), p_{k}\left(x_{t}, y_{t}\right) \in I$ and $k=0, \cdots, N-1, e_{k}$ denotes a line segment defined by two points $p_{k}\left(x_{h}, y_{h}\right)$ (first end point of the $k$ th edge) and $p_{k}\left(x_{t}, y_{t}\right)$ (second end point of the $k$ th edge), and $N$ is the number of edge lines in the set.

\section{ESTIMATION OF CAMERA PARAMETERS}

With roadside or overhead mounted cameras, road images are usually taken in perspective view, in which roads appear as trapeziums and parallel lines appear as converging lines. To utilize the parallel line relationship, we need to restore the image to its original 3-D coordinates, which requires the knowledge of the camera parameters, such as height, tilt angle, and focal length. In theory, camera height may be measured by infrared or laser range sensor. However, commodity sensors' measurement accuracy decreases with measurement distance. In surveillance, the camera may be mounted more than $30 \mathrm{~m}$ above the road surface and some hundreds of meters from the vehicles, in which case sensor measurement may incur many errors. On the other

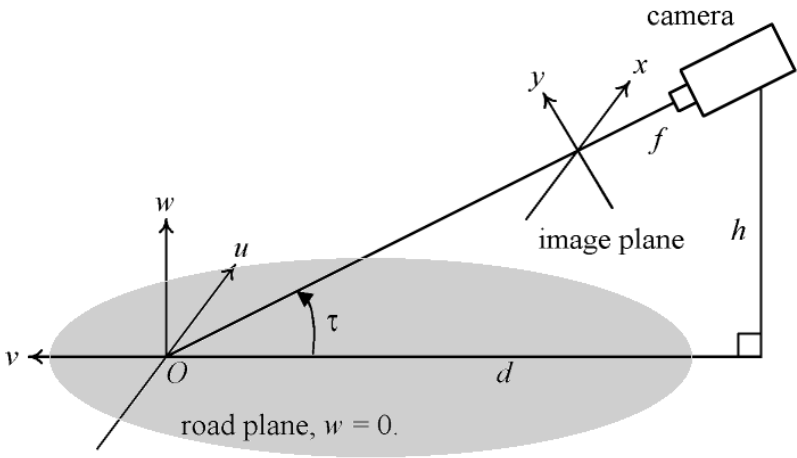

Fig. 4. Camera model.

hand, gimbal angle sensors can be employed to measure the tilt angle too. As its measurement is relative to the horizon instead of the road surface, this introduces error as the road surface may not be parallel to the horizon. To alleviate this problem, it is decided to estimate these parameters using the camera model depicted in Fig. 4 [7], [14], [20]. It describes the transformation of the image coordinates from 2-D to 3-D and vice versa. We term this 2-D-3-D coordinate transformation.

In this model, the road is assumed flat and the camera is mounted at a height of $h$ and focused at $O$, which is on the $w=0$ plane with focal length $f$, while the distance between $O$ and the camera is $d$ and the tilt angle is $\tau$. The transformation, $\Phi$, of a point, $p(x, y)$, from the 2-D image coordinates to a point, $P(u, v 0)$, in the 3-D coordinates may be defined as

$$
P(u, v, 0)=\Phi\{p(x, y)\}
$$

where

$$
\begin{aligned}
& u=x \cdot \sqrt{\frac{(d+v)^{2}+(h-w)^{2}}{f^{2}+y^{2}}} \\
& v=\frac{y \cdot\left[d^{2}+h \cdot(h-w)\right]-f \cdot d \cdot w}{h \cdot f-d \cdot y} .
\end{aligned}
$$

Since the road is on the $w=0$ plane, (3) can be simplified to

$$
\begin{aligned}
& u=\frac{x \cdot h \cdot \sqrt{d^{2}+h^{2}}}{h \cdot f-d \cdot y} \\
& v=\frac{y \cdot\left(d^{2}+h^{2}\right)}{h \cdot f-d \cdot y} .
\end{aligned}
$$

From (4), if the camera parameters $d, h$, and $f$ are known, then every point on the $x-y$ plane has a one-to-one correspondence on the $u-v$ plane. Conversely, if certain feature points have a known relationship on both planes, then the camera parameters may be estimated. Such features can be specific patterns or shapes [21]-[23] intentionally placed on the road or some known line relationships [20]. As placing specific patterns or shapes on the road is rather impractical, the later case presents a possible alternative. However, using a pair of parallel lines (or more) does not provide enough independent equations to solve all three unknowns. In theory, as long as there is a third line that is independent to the parallel line pair, it will enable the unknowns to be found. In practice, the simplest way is to rely on a line perpendicular to the parallel lines. The estimation of the camera parameters is illustrated in the following. 




(a)

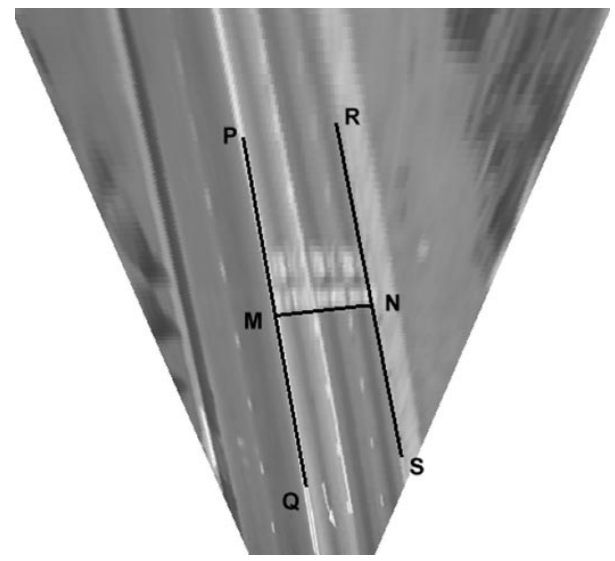

(b)

Fig. 5. Camera parameter estimation. (a) Image on $x-y$ plane. (b) Image on $u-v$ plane.

Consider Fig. 5(a). Let the two parallel lines in 2-D be $p q$ and $r s$, and the perpendicular line be $m n$, where the corresponding lines in 3-D are $P Q, R S$, and $M N$, respectively, as depicted in Fig. 5(b). The point $p$ on the $x-y$ plane is given by $\left(x_{p}, y_{p}\right)$, and the point $P$ on the $u-v$ plane is given by $\left(u_{P}, v_{P}, 0\right)$. In $3-\mathrm{D}$, the slope of $P Q$ is given by

$$
\angle P Q=\frac{v_{P}-v_{Q}}{u_{P}-u_{Q}} .
$$

Substituting (4) into (5), we have

$$
\begin{aligned}
\angle P Q & =\frac{\frac{y_{p}\left(d^{2}+h^{2}\right)}{h f-d y_{p}}-\frac{y_{q}\left(d^{2}+h^{2}\right)}{h f-d y_{q}}}{\frac{x_{p} h \sqrt{d^{2}+h^{2}}}{h f-d y_{p}}-\frac{x_{q} h \sqrt{d^{2}+h^{2}}}{h f-d y_{q}}} \\
= & \frac{f\left(y_{p}-y_{q}\right) \sqrt{d^{2}+h^{2}}}{h f\left(x_{p}-y_{p}\right)-d\left(x_{p} y_{q}-y_{p} x_{q}\right)} \\
= & \frac{Y_{p q} f \sqrt{d^{2}+h^{2}}}{X_{p q} h f-Z_{p q} d}
\end{aligned}
$$

where

$$
\begin{aligned}
Y_{p q} & =y_{p}-y_{q} \\
X_{p q} & =x_{p}-x_{q} \\
Z_{p q} & =x_{p} \cdot y_{q}-x_{p} \cdot x_{q}
\end{aligned}
$$

As lines $P Q$ and $R S$ are parallel and their slopes are identical, therefore

$$
\frac{Y_{p q}}{h \cdot f \cdot X_{p q}-d \cdot Z_{p q}}=\frac{Y_{r s}}{h \cdot f \cdot H_{r s}-d \cdot Z_{r s}} .
$$

From (7), we have

$$
K=f \cdot \frac{h}{d}=\frac{Y_{r s} \cdot Z_{p q}-Y_{p q} \cdot Z_{r s}}{Y_{r s} \cdot X_{p q}-Y_{p q} \cdot X_{r s}} .
$$

The perpendicular line $M N$ is used to establish the following condition:

$$
\angle P Q=\frac{-1}{\angle M N}
$$

Substituting (6) into (9) and expand, we have $f, h$, and $d$ estimated as

$$
\begin{aligned}
& f=K \cdot \sqrt{\left[\frac{\left(Z_{m n}-K \cdot X_{m n}\right) \cdot\left(K \cdot X_{p q}-Z_{p q}\right)}{K^{2} \cdot Y_{m n} \cdot Y_{p q}}-1\right]} \\
& \tau=\tan ^{-1} \frac{h}{d}=\tan ^{-1} \frac{K}{f} .
\end{aligned}
$$

Using these three parameters, Fig. 5(b) depicts the 3-D transformation of Fig. 5(a), viewing from the $z$-axis.

\section{ORIENTATION AND LENGTH DISCRIMINATION}

\section{A. Orientation Discrimination}

Let us first define a road lane to have the following characteristics: locally flat, defined by parallel road markings or curb lengthwise and without sharp bends or roundabouts. Other than those, a road can have multiple lanes. For orientation discrimination, let $\Theta$ define a set of edge lines as follows:

$$
\Theta=\left\{\theta_{k}=\angle e_{k}: \theta_{k} \in[0, \cdots, \pi) \mid k=0, \cdots, N-1\right\}
$$

where $\theta_{k}=\angle e_{k}$ denotes the orientation of the $k$ th edge line on the $u-v$ plane in 3-D and is bounded between 0 and $\pi$. Consider the length of each line $e_{k}$ to be defined by $\left|e_{k}\right|$. For each orientation $\theta_{k}$, we can obtain a length value $L_{\theta_{k}}$, representing the sum of the length of all the edge lines having the same $\theta_{k}$, as given by

$$
L_{\theta_{k}}=\sum\left|e_{i}\right|, \quad \text { for all } \angle e_{i}=\theta_{k} \text {. }
$$

As this is carried out on the $u-v$ plane, parallel lines defining lanes and curbs have large $L_{\theta_{k}}$, while other edge lines have small $L_{\theta_{k}}$. We then apply the $K$-means clustering [24] with $K=2$ to divide $\Theta$ into two sets: $\Theta_{\text {major }}$ and $\Theta_{\text {minor }}$, according to $L_{\theta_{k}}$. The purpose of using the $K$-means in this case is that it identifies a "natural break" in the data set, to partition the data into two sets. The subset $\Theta_{\text {major }}$ contains orientation elements that have large $L_{\theta_{k}}$, meaning that they either have a few long edge lines (curbs, solid lines) or a large number of short edge lines (broken lines). Conversely, $\Theta_{\text {minor }}$ contains orientation elements that have small $L_{\theta_{k}}$ representing minor features in the image. From $\Theta_{\text {major }}$, we may define a new set of edge lines by

$$
E_{\Theta}=\left\{e_{k} \in E: \theta_{k} \in \Theta_{\text {major }}\right\} \text {. }
$$

Fig. 6 depicts the resulting edge maps of Fig. 3(b) in $x-y$ and $u-v$ planes after orientation discrimination. It can be seen that most edges due to roadside objects, uneven road colors, and 

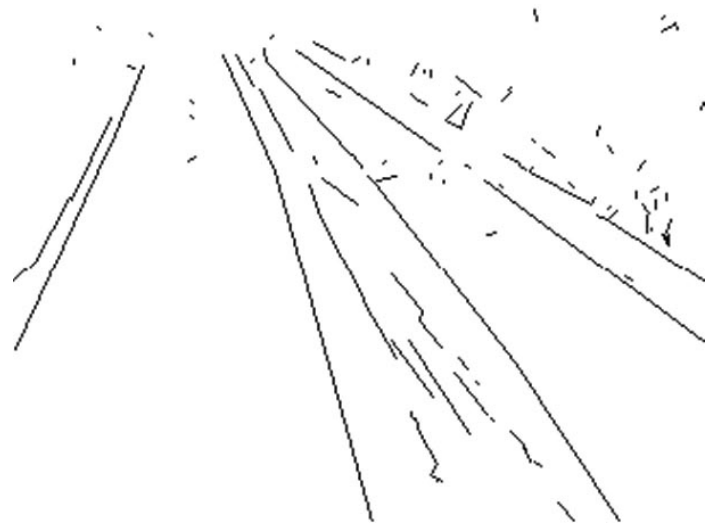

(a)

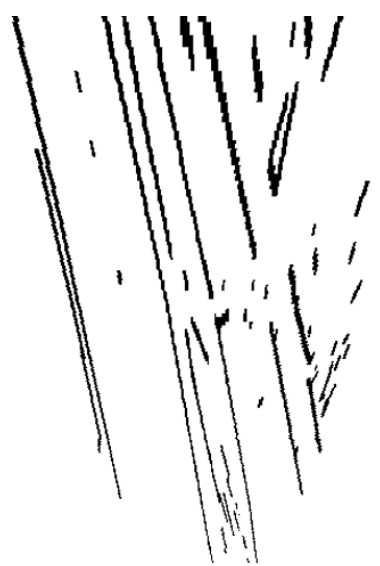

(b)

Fig. 6. Orientation discrimination result (city road). (a) Edge map on $x-y$ plane. (b) Edge map on $u-v$ plane.

painted instructions have been eliminated. It should be noted that the short broken lines dividing two lanes have also been removed.

\section{B. Length Discrimination}

As also noted in Fig. 6, some of the remaining short and isolated edge lines have been wrongly clustered to $\Theta_{\text {major }}$. To further eliminate those lines, the edge lines in $E_{\Theta}$ are first linked according to their orientations and locations. The link conditions are set as follows [25], [26]:

$$
\left|\theta_{i}-\theta_{j}\right|<\varepsilon_{1}, \quad \text { and }\left|e_{i}-e_{j}\right|<\varepsilon_{2}
$$

where $e_{i}, e_{j} \in E_{\Theta}$, and $\theta_{i}, \theta_{j}$ are their orientation, respectively. Both $\varepsilon_{1}$ and $\varepsilon_{2}$ are predefined limits. If two edge lines satisfy the above conditions, they are connected as given by (16), and Fig. 7 depicts the connected edge map of Fig. 6:

$$
\bar{e}_{k}=\left\{\bar{p}_{k}\left(x_{k, h}, y_{k, h}\right), \bar{p}_{k}\left(x_{k, t}, y_{k, t}\right)\right\}
$$

where

$$
\bar{p}_{k}\left(x_{k, h}, y_{k, h}\right)=\max _{y}\left\{p_{i}\left(x_{i, h}, y_{i, h}\right), p_{j}\left(x_{j, h}, y_{j, h}\right)\right\}
$$

and

$$
\bar{p}_{k}\left(x_{k, t}, y_{k, t}\right)=\min _{y}\left\{p_{i}\left(x_{i, t}, y_{i, t}\right), p_{j}\left(x_{j, t}, y_{j, t}\right)\right\}
$$

As can be seen in Fig. 7, the edge map after edge linking still contains a number of short and isolated lines. This problem can

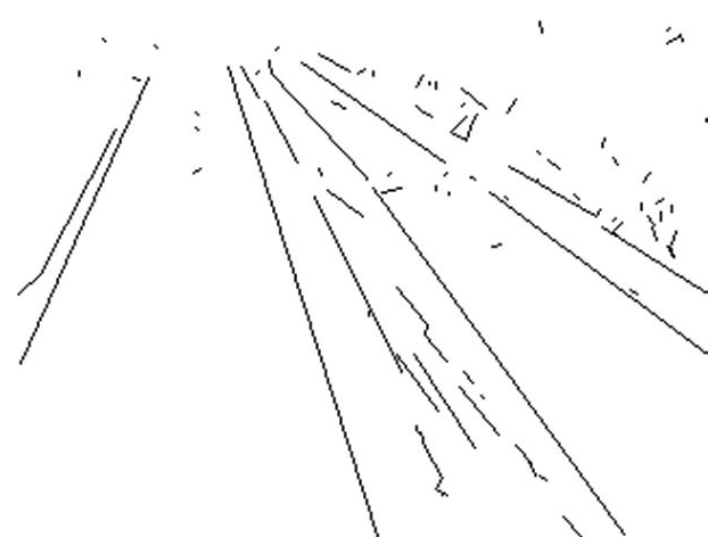

(a)

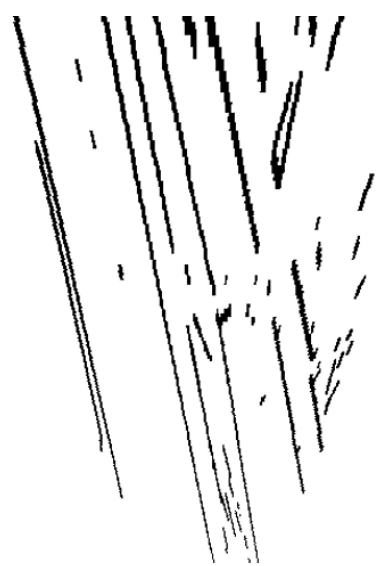

(b)

Fig. 7. Edge linking result (city road). (a) Edge map on $x-y$ plane. (b) Edge map on $u-v$ plane.

be dealt with by length discrimination. First, let us define the set of connected edge lines by

$$
L=\left\{\left|\bar{e}_{k}\right| \mid k=0, \cdots, M-1\right\} .
$$

Again, we apply $K$-means clustering with $K=2$ to decompose the edges segments into two sets: $L_{\text {long }}$ and $L_{\text {short }}$ according to $\left|\bar{e}_{k}\right|$. In this case, only $L_{\text {long }}$ is retained, with $L_{\text {short }}$ discarded. The final set of edge lines can be described by (18) and the corresponding edge maps are depicted in Fig. 8:

$$
L_{e}=\left\{\bar{e}_{k}:\left|\bar{e}_{k}\right| \in L_{\text {long }}\right\} \text {. }
$$

From Fig. 8, it can be seen that almost all the short and isolated edges have been eliminated through length discrimination. The remaining edge lines correspond to long parallel lines that define the lane and curb positions. It should also be noted that the lines due to the arrow remain, while the curb on the left is now represented by two lines instead of one. These lines will be eliminated during lane analysis.

\section{LANE ANALYSIS}

In general, a road lane consists of two parallel edge lines with an almost constant perpendicular distance between them. As depicted in Fig. 8(b), after the discrimination of orientation and length, all the lines are almost parallel to each other. To determine which pair of lines defines a lane, let the perpendicular distance, $d_{i k}$, between a parallel edge line pair, $\left\{\bar{e}_{i}, \bar{e}_{k}\right\}$, be

$$
d_{i k}=\left|\bar{e}_{i}, \bar{e}_{k}\right| \sin \left(\theta_{i}-\tan ^{-1} \angle\left(\bar{e}_{i}, \bar{e}_{k}\right)\right)
$$



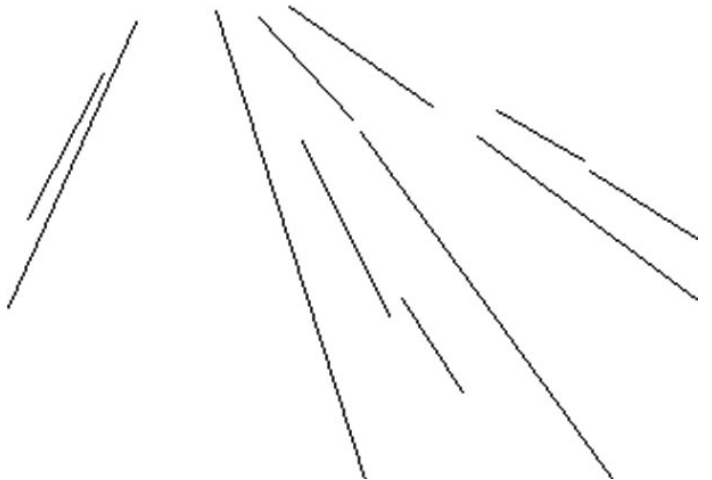

(a)

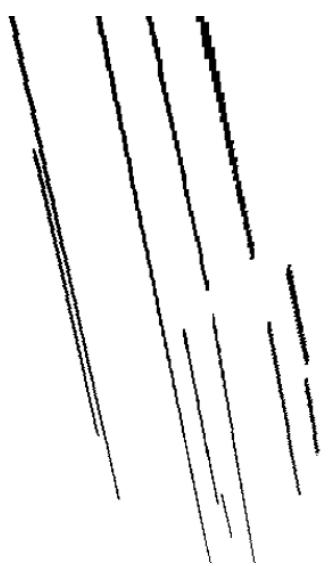

(b)

Fig. 8. Length discrimination result (city road). (a) Edge map on $x-y$ plane (b) Edge map on $u-v$ plane.

where

$$
\left|\bar{e}_{i}, \bar{e}_{k}\right|=\sqrt{\left(x_{p_{i, t}}-x_{p_{k, t}}\right)^{2}+\left(y_{p_{i, t}}-y_{p_{k, t}}\right)^{2}}
$$

and

$$
\angle\left(\bar{e}_{i}, \bar{e}_{k}\right)=\frac{y_{p_{i, t}}-y_{p_{k, t}}}{. x_{p_{i, t}}-x_{p_{k, t}}} .
$$

If $d_{i k}$ is similar to $W$, a predefined lane width, a single lane is detected. If $d_{i k}$ is smaller than $W$, then there is no lane between the pair. If $d_{i k}$ is larger than $W$, there is possibly more than one lane between the pair, and the number of lanes, $N_{l}$ can be estimated by

$$
N_{l}=\left\lfloor\frac{d_{i k}}{W}\right\rfloor
$$

To estimate the rest of the lanes, the other edge lines in $L_{e}$ are evaluated. Those edge lines that do not belong to a pair forming one or more lanes are discarded at the end.

Finally, the center line of each lane is calculated as the arithmetic mean of the two lines defining a lane. These lines could be edge lines or deduced from (20). Fig. 9(a) depicts the center lines calculated from Fig. 8(b) and Fig. 9(b) depicts the center lines overlaid onto the background image. It can be observed that the center lines are reasonably accurate, particularly for those lanes nearer to the camera.
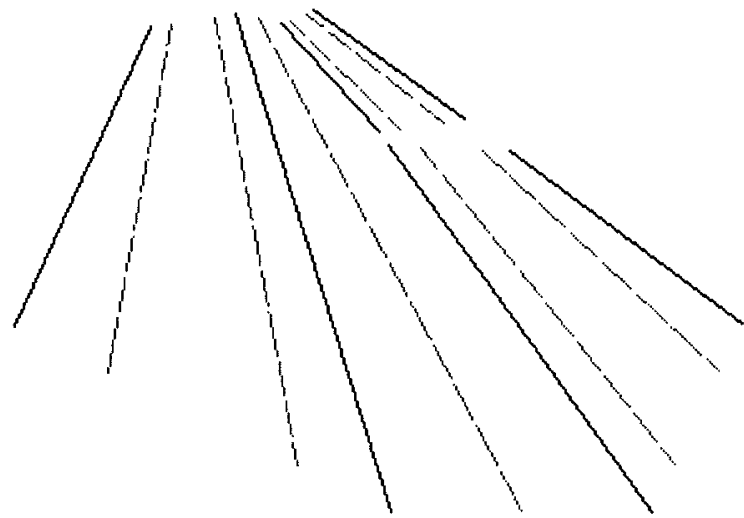

(a)

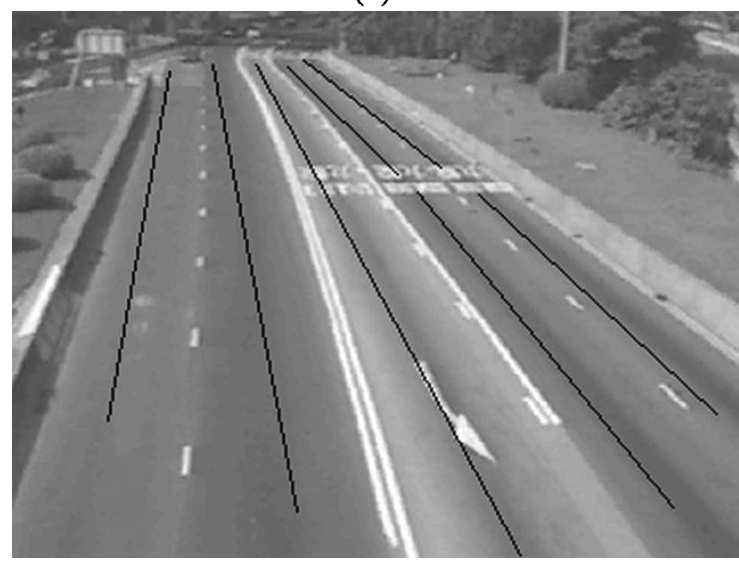

(b)

Fig. 9. Lane analysis result (city road). (a) Broken lines showing the center lines. (b) Center lines overlaid on the background image.

\section{Practical Evaluation}

To further illustrate the effectiveness of the lane detection algorithm, a practical multilane freeway scenario was used for evaluation. The original traffic sequence was supplied by the Transport Department of the Hong Kong SAR government, from one of their overhead mounted CCTV cameras situated at a busy freeway in Hong Kong. The image as depicted in Fig. 10 was background extracted from less than 30 frames $(<1 \mathrm{~s})$ of the video that contains a few moving vehicles. The image shows an eight-lane freeway with four lanes in each direction, together with an entrance lane on the left and an exit lane on the right. Lane markings and curbs are reasonably clear, although they suffer from blurring. Roadside objects such as containers, bushes, and other roads below are also visible. In line with normal practice, each frame is stamped with date, time, and a camera identification code for reference purposes. Since the background estimation was done in less than $1 \mathrm{~s}$, the date/time stamps appear to be stationary and, therefore, not removed from the image. They present no major problem as they will be eliminated later by the discrimination processes. As a whole, the image contrast and resolution are poor, and there are intensity and spatial variations on the road such as broken central partition and different surface colors.

\section{A. Edge Detection and Line Approximation}

The thinned edge map using the Sobel edge detection is depicted in Fig. 11(a). The approximated edge map is shown in 


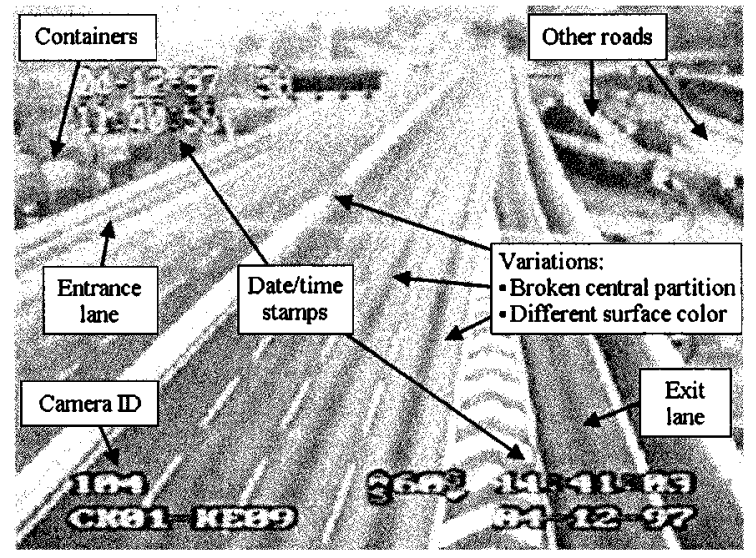

Fig. 10. Freeway background image.

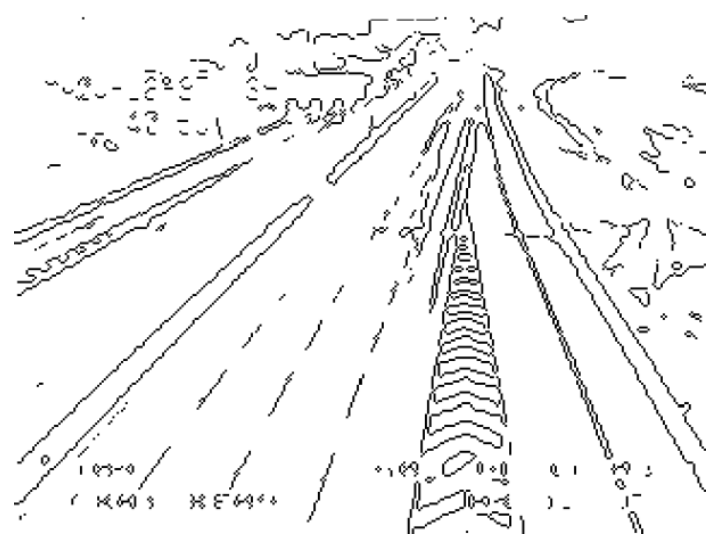

(a)

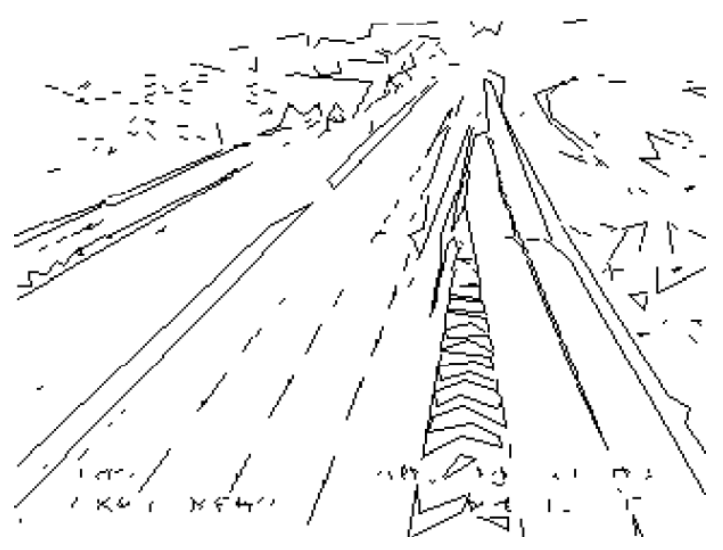

(b)

Fig. 11. Thinned and approximated edge maps (freeway). (a) Thinned edge map. (b) Line approximated edge map.

Fig. 11(b), with a maximum deviation of two pixels. It can be seen from Fig. 11(a) that the thinned edge map is basically useless as it is. Although the strong features of the central partition, the curbs, the lane split markings, and some of the lane markings are evident, there are numerous short and disjointed edges representing some of the weak. It is also noted that the intensity variations on the road and the information printed on the image introduce substantial interference to the resulting edge map. From Fig. 11(b), the line approximated edge map is a slight improvement from Fig. 11(a), where edge lines are smoother. However, further processing is clearly needed.

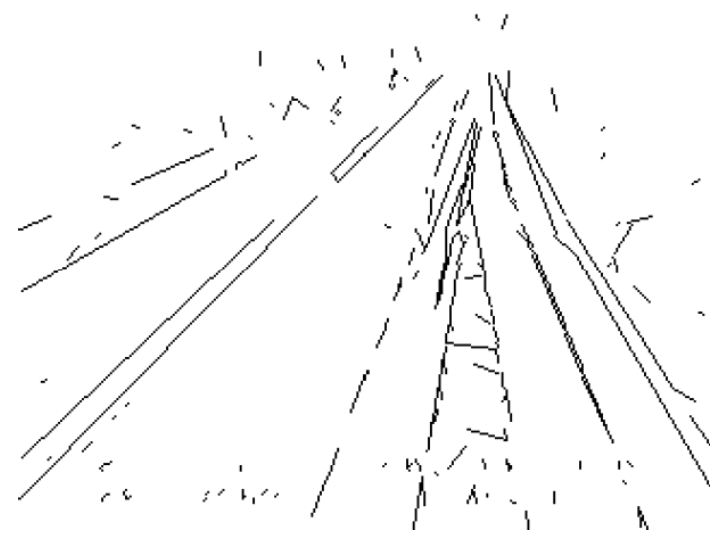

Fig. 12. Orientation discrimination result (freeway).

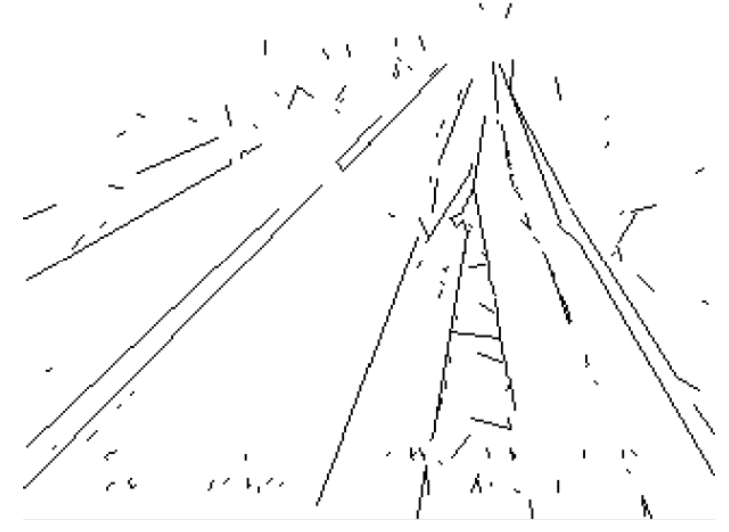

Fig. 13. Edge linking result (freeway).

\section{B. Orientation Discrimination}

The result of orientation discrimination is depicted in Fig. 12. Comparing Fig. 12 with Fig. 11(b), it can be observed that edge lines that fall into the minor orientation set are mainly those short lines with orientations quite different from those of the majority of lines. These include the cluster of short lines on the top left-hand corner and top right-hand corner of the image, the printed characters, as well as the broken lines for lane separation and the lane markings associated with the entrance and exit lanes. The removal of some of the broken lines defining the lane separation is undesirable as the lanes have now merged and some of the strong features such as the curb on the left are no longer so well defined. Other than that, the removal of the features associated with the road splitting, roadside objects, and printed characters on the image are welcome. On the other hand, it is clear that there are still unwanted edges at various orientations left on the edge map.

\section{Length Discrimination}

Fig. 13 depicts the edge map after line linking. The effect of line linking is well illustrated by the linking of the broken line at the center of the map into a solid long line. Other than that, the linked edge map is little different from Fig. 12. On the other hand, the edge map after length discrimination is much different from Fig. 13. As depicted in Fig. 14, a large number of short-edge lines have been eliminated from the edge map. Even though some of the lines such as those on the far left of the 


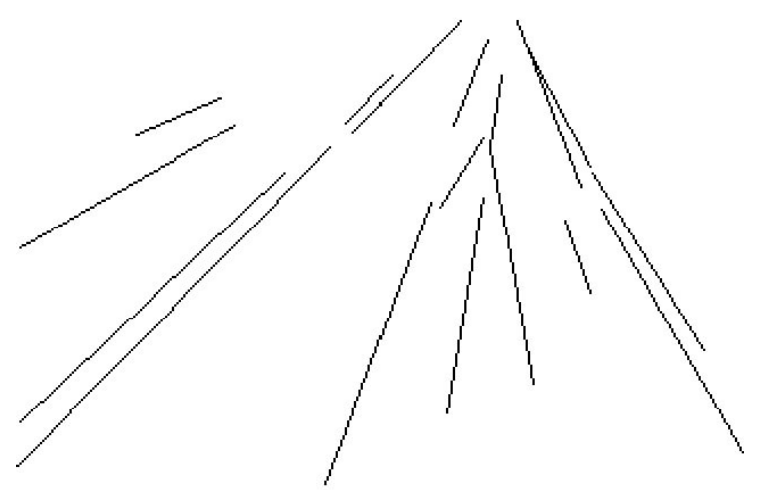

Fig. 14. Length discrimination result (freeway).

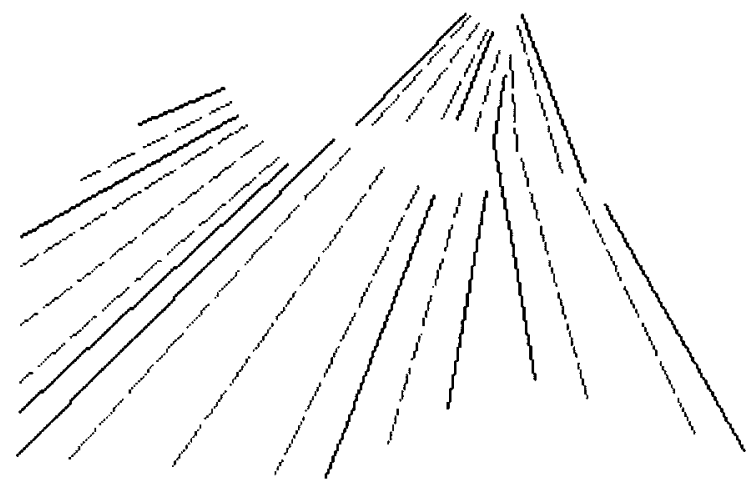

(a)

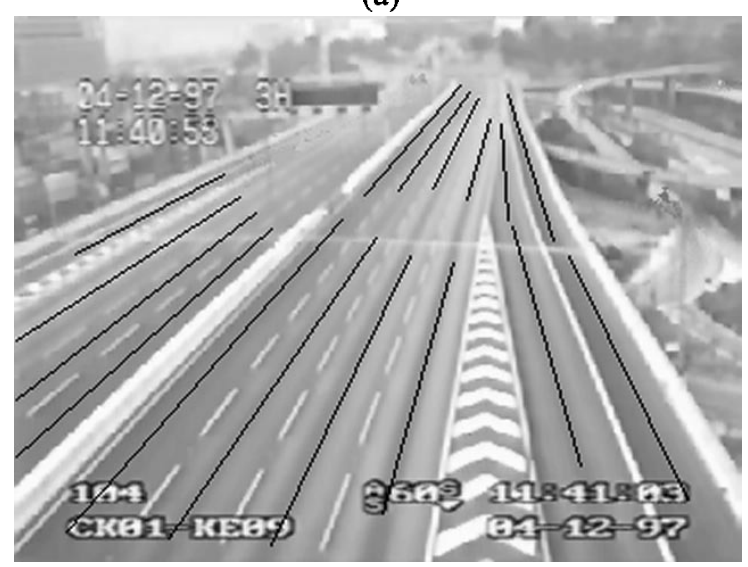

(b)

Fig. 15. Lane analysis result (freeway). (a) Broken lines showing the center lines. (b) Center lines overlaid on the background image.

image should be retained, the remaining lines in the edge map are sufficient to define the lanes nearer to the camera, including the entrance and exit lanes. It is noted that the far view of the road is poorly detected, while the near view is well detected. This is expected and considered acceptable as the near view lane information is far more important than the far view.

\section{Lane Analysis}

Finally, center lines of the lanes are calculated from the edge lines in Fig. 14. Each adjacent line pair is examined, and those that cannot be paired up are discarded. The width of the parallel line pairs is analyzed and based on a presumed width of $4 \mathrm{~m}$ per lane; the resulting center lines for the image are depicted as broken lines on Fig. 15(a), and they are overlaid on the background image in Fig. 15(b) for inspection. From Fig. 15(b), it can be seen that the center lines obtained are acceptable. It is also interesting to note that due to the intensity/spatial variations, the center lines are broken up into two sets for the six lanes on the right-hand side. This is probably due to the broken central partition and the exit lane. These broken lines may be merged to form complete center lines with additional analysis. As the camera was looking down these lanes, these center lines extended reasonably far down the road. However, on the left-hand four lanes and entrance lane, only the near view center lines have been detected, and their accuracy is debatable. As visual traffic surveillance focuses on unidirectional traffic, this effect is probably tolerable in real application.

\section{CONCLUSIONS}

We have presented a lane detection algorithm that can effectively detect the center lines of multiple lanes under normal and practical freeway conditions. The crux of the algorithm is in the discrimination of edge line orientation and length in 3-D. Its first step relies on traditional edge detection, thinning, and straight-line approximation. After that, the edge lines are transformed into 3-D based on estimated camera parameters. As roads and lanes usually have long parallel line features, the 2-D to 3-D transformation helps the orientation discrimination to eliminate lines with orientations that are not shared by many. Moreover, the length of curbs and lanes assist in discriminating short lines due to other features. As $K$-means clustering is used for the discrimination in both cases, the algorithm is generally robust. This algorithm was further evaluated using a practical multilane freeway sequence, which contains spatial and color irregularities. Our evaluation shows that it is indeed able to extract the required information with both performance and errors maintained at an acceptable level, using just one frame of the sequence. As it is, the algorithm can potentially be applied to many visual traffic surveillance problems such as vehicle tracking, red light runner detection, incident detection, etc.

\section{ACKNOWLEDGMENT}

The authors thank the Transportation Department of the Government of Hong Kong SAR for allowing access to their freeway traffic management and control system and for the use of some of their images for research.

\section{REFERENCES}

[1] Intelligent Transport Systems Handbook in Japan, Japan: Highway Industry Development Organization, 1997.

[2] J. Palen, "The need for surveillance in Intelligent Transportation Systems," Intellimotion, vol. 6, no. 1, pp. 1-10, 1997.

[3] M.-P. D. Jolly, S. Lakshmanan, and A. K. Jain, "Vehicle segmentation and classification using deformable templates," IEEE Trans. Pattern Anal. Machine Intell., vol. 18, pp. 293-308, Mar. 1996.

[4] F. Bartolini, V. Cappellini, and C. Giani, "Motion estimation and tracking for urban traffic monitoring," Proc. IEEE IICIP '96, vol. 3, pp. 787-790, 1996.

[5] B. Gloyer, H. K. Aghajan, K. Y. Siu, and T. Kailath, "Video-based freeway monitoring system using recursive vehicle tracking," in Proc. SPIE, vol. 2421, 1995, pp. 173-180. 
[6] A. H. S. Lai and N. H. C. Yung, "A system architecture for visual traffic surveillance," in 5th World Congr. ITS, Korea, 1998.

[7] L. T. Schaaser and B. T. Thomas, "Finding road lane boundaries for vision-guided vehicle navigation," in Vision-Based Vehicle Guidance. New York: Springer-Verlag, 1992, pp. 238-254.

[8] T. M. Jochem and S. Baluja, "A massively parallel road follower," in Proc. Computer Architectures for Machine Perception, 1993.

[9] D. Frank, "Road markings recognition," in Proc. IEEE ICIP '96, vol. II, Lausanne, Switzerland, 1996, pp. 669-672.

[10] P. Charbonnier, F. Diebolt, Y. Guillard, and F. Peyret, "Road markings recognition using image processing," in Proc. ITSC, 1997.

[11] C. Thorpe, M. H. Hebert, T. Kanada, and S. A. Shafer, "Vision and navigation for the Carnegie-Mellon Navlab," IEEE Trans. Pattern Anal. Machine Intell., vol. 10, no. 3, pp. 362-373, 1988.

[12] W. Kasprzak, H. Niemann, and D. Wetzel, "Adaptive road parameter estimation in monocular image sequences," in Proc. Brit. Machine Vision Conf. 1994, 1994, pp. 691-700.

[13] Y. L. Tang and R. Kasturi, "Runway detection in an image sequence," in Proc. SPIE, vol. 2421, 1995, pp. 181-190.

[14] N. W. Campbell and B. T. Thomas, "Lane boundary tracking for an autonomous road vehicle," in Proc. Brit. Machine Vision Conf. '92, 1992, pp. 157-166.

[15] K. Kluge and S. Lakshmanan, "Lane boundary detection using deformable templates: Effects of image subsampling on detected lane edges," in Proc. 2nd Asian Conf. Computer Vision 1995, Singapore, 1995, pp. 329-339.

[16] N. H. C. Yung and A. H. S. Lai, "A fast and accurate scoreboard algorithm for estimating stationary backgrounds in an image sequence," in Proc. IEEE ISCAS '98, 1998, Catalog No. 98CH36187, ISBN: 0-78034458-8, TPA13-3.

[17] N. H. C. Yung and A. H. S. Lai, "Performance evaluation of a feature-preserving filtering algorithm for removing additive random noise in digital images," SPIE J. Opt. Eng., vol. 35, no. 7, pp. 1871-1887, 1996.

[18] A. H. S. Lai and N. H. C. Yung, "A new feature preserving filter algorithm based on a prior knowledge of pixel types," SPIE J. Opt. Eng., vol. 35 , no. 12 , pp. 3508-3521, 1996.

[19] R. C. Gonzalez and R. E. Woods, Digital Image Processing. Reading, MA: Addison-Wesley, 1992, ch. 7, pp. 416-428.

[20] E. K. Bas and J. D. Crisman, "An easy to install camera calibration for traffic monitoring," in Proc. ITSC, 1997.

[21] L. L. Wang and W. H. Tsai, "Camera calibration by vanishing lines for 3-D computer vision," IEEE Trans. Pattern Anal. Machine Intell., vol. 13, no. 4, pp. 370-376, 1991

[22] E. A. Parrish, Jr. and A. K. Goksel, "A camera model for natural scene processing," Pattern Recognition, vol. 9, pp. 131-136, 1977.

[23] Y. Nakazawa, T. Komatsu, and T. Saito, "A simple cue-based method for camera calibration and 3-D shape measurement with a single moving camera," Proc. IEEE ICIP'96, vol. 2, pp. 293-296, 1996.
[24] J. A. Hartigan, Clustering Algorithms. Toronto, ON, Canada: Wiley, 1975

[25] G. W. Cook and E. J. Delp, "Multiresolution sequential edge linking," Proc. IEEE ICIP'95, vol. 1, pp. 41-44, 1995.

[26] S. Vasudevan, R. L. Cannon, and J. C. Bezdek, "Heuristics for intermediate level road finding algorithms," Int. J. Comput. Vis., Graph., Image Process., vol. 44, pp. 175-190, 1998.

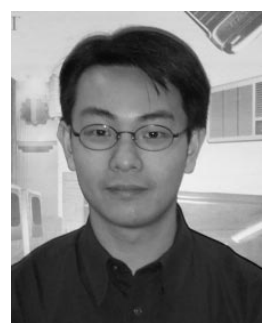

Andrew H. S. Lai (S'89-S'93-M'95) received the B.Eng. degree from the University of Hong Kong, Pokfulam, Hong Kong, in 1994, the M.Sc. degree from the University of Surrey, Surrey, U.K., in 1995, and the Ph.D. degree from the University of Hong Kong in 2000 .

He is currently an Honorary Assistant Professor at the University of Hong Kong and Senior Research Engineer at the Laboratory for Intelligent Transportation Systems Research. His research interest includes visual traffic surveillance, digital image processing, and wireless data communication.

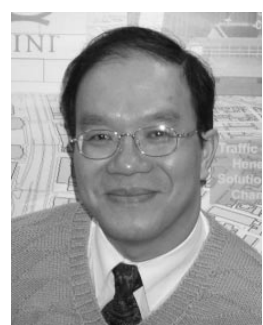

Nelson H. C. Yung (S'82-M'85-SM'96) received the B.Sc. and Ph.D. degrees from the University of Newcastle upon Tyne, Newcastle upon Tyne, U.K., in 1982 and 1985 , respectively.

He was Lecturer at the University of Newcastle upon Tyne from 1985 to 1990, where he was involved in the research and development $(\mathrm{R} \& \mathrm{D})$ of digital image processing and parallel processing. From 1990 to 1993, he was Senior Research Scientist at the Department of Defence, Australia, where he headed a team on the R\&D of military-grade signal analysis systems. He joined the University of Hong Kong, Pokfulam, Hong Kong, in 1993 as Associate Professor. He leads a research group in digital image processing and intelligent transportation systems. He is the founding Director of the Laboratory for Intelligent Transportation Systems Research, and has published more than 90 research papers. He is a reviewer for Institute of Electrical Engineers (IEE) Pt. G, SPIE Optical Engineering, HKIE Proceedings, and Microprocessors and Microsystems Journal.

Dr. Yung is a Chartered Electrical Engineer and Member of the HKIE and IEE. His biography is published in Who's Who in the World. He serves as reviewer for the IEEE TRANSACTIONS ON SYSTEMS, MAN, AND CYBERNETICS and IEEE TRANSACTIONS ON Signal PROCESSING. 